\title{
AEAR SURFACE - GEOSCIENCE?
}

\section{The potential of electrical imaging for field root zone phenotyping}

\section{Introduction}

Providing enough food for a growing population while preserving natural resources and biodiversity is one of the challenges of the 21st century. A key pathway to maximize yields in a sustainable way is to select and grow crops that are optimally adapted to their environment. Crops with greater yield in infertile soils will substantially improve the productivity and sustainability of low-input agroecosystems. In high-input agroecosystems, they will reduce the environmental impacts through the reduction of irrigation and fertilisation needs. Plant performance is determined by characteristics or 'traits' which are partially genetically determined. There are several tools to identify genes attached to interesting plant traits that could be targeted by breeders. Genome-wide association studies (GWAS), for example, screen a set of genetic variants of the same plant in different individuals to see if any variant is systematically associated with a desired trait.

Nevertheless, cultivars with the same genome (G) express different appearances in different environments (E) and under different management practices (M). This GxExM interaction results in a range of 'phenomes' for the same genetic individual in different environments. Already at regional scale, pedo-climatic conditions and management practices vary significantly. In addition, climate is changing and the field conditions of today will not be those of tomorrow. Breeders are therefore not only interested in looking at plant characteristics in laboratories or controlled greenhouses, but are increasingly aware of the need to test cultivars in a relevant environment, i.e. field conditions, before going to the market. Phenotyping, or the practice of characterizing how genetic characteristics are expressed as plant traits in various spatially and temporally dynamic environments, has therefore become the foundation of breeding practice (Costa et al. 2019).

A phenome can include diverse structural and functional aspects or traits, like plant biomass, root morphology and function, leaf characteristics, fruit traits, but also chemical substances with roles in plant defense and biosphere-atmosphere interactions and microbiome-plant interactions. Functional trait concepts can also be included, like photosynthetic efficiency and (a)biotic stress resistance/tolerance. Above-ground traits are already extensively studied in lab and field, especially with the rise of drone technology monitoring canopy traits (e.g. high-throughput field phenotyping (HTFP) using different camera systems (RGB, multispectral, hyperspectral, thermal, LIDAR, ... ) (Xie and Yang 2020). Dedicated high-throughput platforms allow for efficient screening of a high number of individuals with high spatial and temporal resolution.

Phenotyping the below-ground traits is less straightforward, due to the opaque nature of soil. To date, classical non-destructive 2D techniques, such as agar plates or rhizotrons, have been driving our understanding of root development. Pouch systems growing seedlings on germination paper are also widely used, since they can be deployed in high-throughput phenotyping systems. Non-destructive analysis of 3D root growth is possible using transparent gels or hydroponic conditions, but results are often difficult to extrapolate to more mature plants and to field conditions (Atkinson et al. 2019). 3D phenotyping of roots in soil is currently done with X-ray computed tomography (X-ray CT), magnetic resonance imaging (MRI) and position emission tomography (PET). Nevertheless, this is either with young, small plants (non-destructive) or under field conditions (destructive). Shovelomics, or root crown phenotyping, is one of the most widely adopted high-throughput field methods for below-ground phenotyping. However, the process remains destructive and is very labour-intensive. At the european level, a network of in- and outdoor phenotyping platforms is being established to improve the access to and collaboration between existing infrastructures: EMPHASIS, as a follow-up of the European Plant Phenotyping Network (EPPN2020).

Non-invasive geophysical techniques to study the root system and soil profile have substantially advanced in recent years (Ehosioke et al. 2020). Only a few techniques can actually retrieve direct information on the root system architecture (e.g. ground-penetrating radar (GPR) for coarse roots ) or root biomass density (e.g. electrical impedance tomography (EIT)). Nevertheless, the biggest potential of geophysical techniques lies in their indirect monitoring of water depletion or other changes in the root zone, especially when they are deployed in time-lapse mode. Electro-magnetic induction (EMI) 


\section{NEAR SURFACE $\triangle$ GEOSCIENCE}

and electrical resistivity tomography (ERT), are the techniques which have been most implemented in the field for phenotyping purposes. EMI is less effective in drier soils than ERT, nevertheless can be used to rapidly screen large populations for differences in root activity

\section{Case-study: the EMPHASIS-BELGIUM field phenotyping installation under rain-out shelters}

\section{Material and methods}

Figure 1 shows the general workflow to investigate the performance of different electrode setups for electrical resistivity tomography (ERT) for phenotyping purposes. We first generated a range scenarios of soil moisture dynamics for different soils and grass cultivars (a). For selected days, we converted the soil moisture distribution to an electrical conductivity distribution using petrophysical models from literature (b). Then we generated ERT measurements from these distributions for selected electrode setups (c). We added $2 \%$ noise to these measurements and then inverted them back to obtain ERTderived conductivity distributions (d). In this way, we can check the performance of different electrode arrays to delineate root water uptake patterns in various realistic conditions.

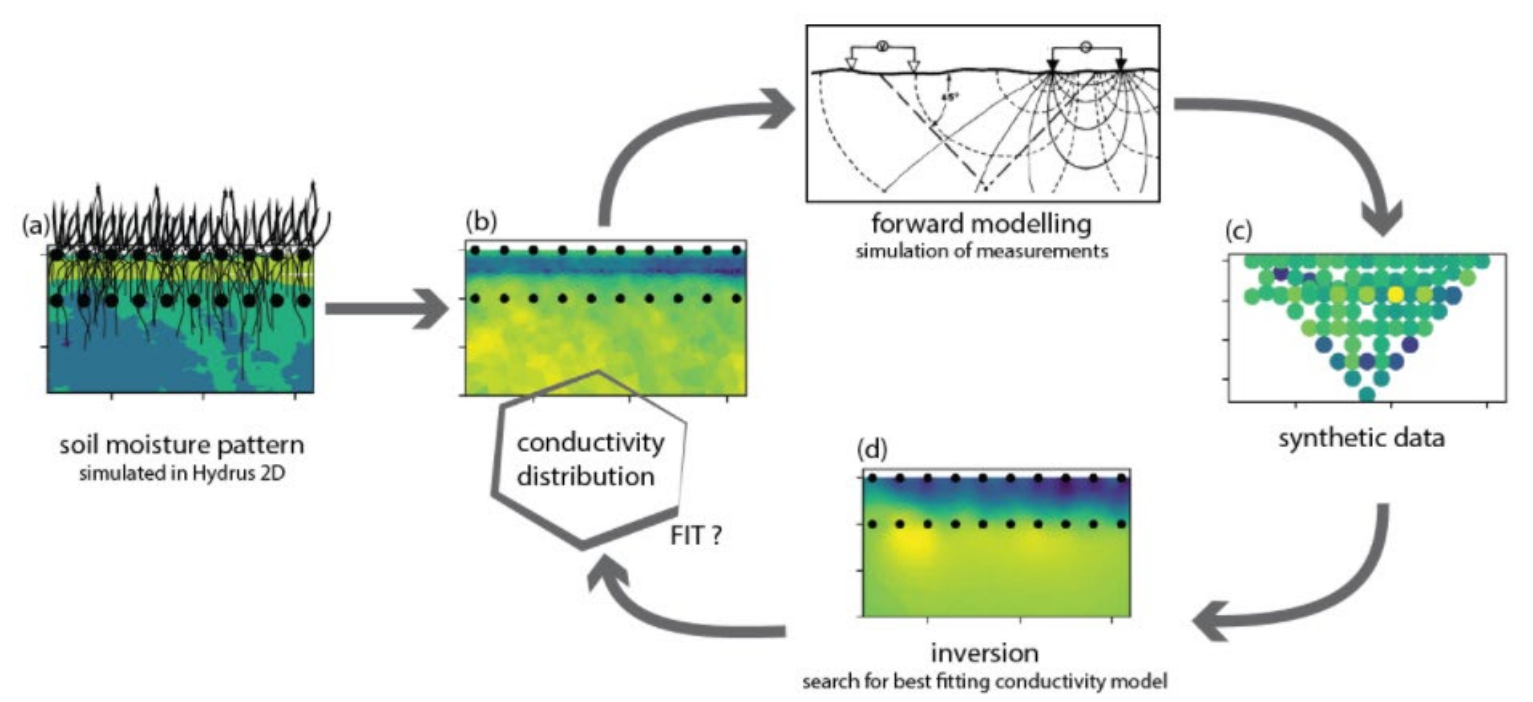

Figure 1 Schematic overview of workflow to test the performance of different electrode arrays for root zone phenotyping using virtual experiments.

We constructed a hydrological model using Hydrus2D/3D (Šimůnek et al., 1996) which allows simulating soil water and root water uptake fluxes. The hydrological simulation domain was $5 \mathrm{~m}$ wide and $2 \mathrm{~m}$ deep within which we located an experimental plot of $1.5 \mathrm{~m}$ with and $1 \mathrm{~m}$ depth. We considered a soil profile with 2 horizons. The top $0-20 \mathrm{~cm}$ remained the same in all scenarios (silt loam), whereas the next horizon varied: sandy loam (S), loam (L) and clayey loam (C). The hydraulic parameters for the simulations were taken from the Rosetta catalogue within Hydrus 2D/3D. We implemented a $1 \mathrm{D}$ root profile of pasture according to the Vrugt model and varied the maximum rooting depth (30-100 $\mathrm{cm})$ and depth of maximum uptake $(20-30 \mathrm{~cm})$ intensity to represent 5 virtual cultivars of the same plant. We ignored the heterogeneity of the third dimension. We used interpolated weather data of the Belgian Royal Meteorological Service (KMI-IRM) for Melle, Belgium from 2018 to generate rainfall and evapotranspiration rates. January until May 2018 served as a spin-up period for the hydrological model. We selected day of the year (DOY) 152 as reference date and 159, 182 and 212 as examples of increasing soil drying after 1 week, 1 month and 2 months, respectively.

The water content distributions for the three selected soil types and on the 4 selected DOYs were subsequently converted into bulk electrical conductivity using petrophysical relationships of similar soils published in the literature. We applied one petrophysical 


\section{CONFERENCE \& EXHIBIIION NEAR SURFACE GEOSCIENCE'21}

relationship per soil profile, so the same for both horizons, which is a simplification of reality.

Electrical resistivity tomography data are voltages measured over 'receiver' electrode pairs for known injected currents over 'source' electrode pairs. The governing equation for DC resistivity is

$$
\nabla \cdot(-\sigma \nabla \Phi)=I\left(\delta\left(\vec{r}-\overrightarrow{r_{s^{+}}}\right)-\delta\left(\vec{r}-\overrightarrow{r_{s^{-}}}\right)\right)
$$

where $\sigma$ is the electrical conductivity (i.e. the inverse of the resistivity), $\Phi$ is the electric potential, I is the input current at the positive and negative dipole locations (r), captured as Dirac delta functions, $\delta$. We simulated four electrode setups: transect with surface electrodes (TRAN), transect with surface and buried electrodes (TRAN BUR), transect with surface electrodes combined with 2 borehole electrode sticks (TRAN_BORE) and only borehole electrode sticks (BORE). We considered that an experimental phenotyping plot is $1.5 \mathrm{~m}$ wide, resulting in a transect of 10 electrodes with $30 \mathrm{~cm}$ spacing of which 6 electrodes are located inside the plot. The buried electrodes are located at $50 \mathrm{~cm}$ depth. The borehole sticks contain electrode rings every $20 \mathrm{~cm}$. All electrodes were simulated as point electrodes, which is a simplification of reality, especially considering the small electrode spacing. We added $2 \%$ Gaussian noise to the generated synthetic data to represent data and model uncertainty. The measurements are composed of a set of transfer resistances (R) from different four electrode configurations (quadrupoles).

The translation of geoelectrical measurements to geoelectrical properties requires the use of inverse methods. These methods aim to find the best distribution of geoelectrical parameters that is consistent with observed measurements. This involves minimizing the misfit between the set of quadrupole measurements and the predicted response from a geoelectrical model.

$\varphi(m)=\varphi_{d}(m)+\beta \cdot \varphi_{m}(m)$,

where the objective function for resistivity model $\varphi$ is the sum of the data functional $\varphi_{\mathrm{d}}$ and the model functional $\varphi_{\mathrm{m}}$ containing smallness and smoothness penalties multiplied by the regularization factor $\beta$. $\mathrm{m}$ is the vector of model parameters, in this case the resistivity values. Because of the non-linear nature of the problem, the inversion proceeds in an iterative manner until the misfit between the predicted response and the measurements are within a given tolerance. Therefore, the inversion seeks to find the resistivity distribution that can explain the measurements to an acceptable extent. For more details about the inverse methods used here, see Binley and Kemna (2005) and the Jupyter notebook associated to this extended abstract.

\section{Preliminary results of simulations}

Figure 2 shows the results of the different electrode configurations for a loamy soil for four selected DOY. The effect of the drying of the upper soil layers is visible on the first row as the DOY increased. After translation of this soil moisture to resistivity (here shown for the loamy soil), forward modelling and inversion of the results, the recovered resistivity distributions is showed in the other rows. The TRAN arrays only recover very partial pattern due to its limited depth of investigation. The TRAN_BUR does better, especially at recovering the lateral extend of the boundary. The TRAN_BORE and BORE case both recover well the interface with some slight artefacts close to the boreholes, probably due to the sensitivity distributions. This effect seems larger for the BOR than for the TRAN_BOR case. The virtual experiment clearly shows that a transect with only surface electrodes (blue line) fails to capture soil moisture changes below ca. $30 \mathrm{~cm}$ in most cases. Due to the lack of sensitivity, the inverted models are mainly determined by the starting model and not by the data. The surface transect combined with borehole electrodes at the plot boundaries performs best to retrieve the shape of the soil moisture depletion pattern, especially close to the surface, where the other methods have more difficulties. The transect with buried electrodes performs well, with often a good retrieval of the magnitude of the depletion pattern peak. Of all tested electrode arrays, the combination of a surface transect with borehole electrodes (TRAN BORE) works best, closely followed by the transect combined with buried electrodes (TRAN_BUR). Surface electrodes only is not sufficient to use electrical imaging as phenotyping tool. Next to electrode setup, also an optimization of the electrode array should be considered in order to optimize the sensitivity and duration of the measurement. 


\section{( NEAR SURFACE - GEOSCIENCE}
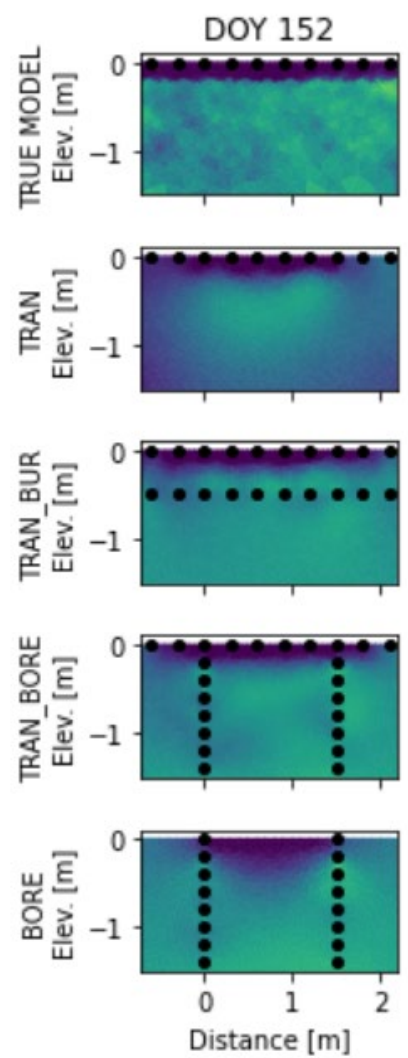

DOY 159
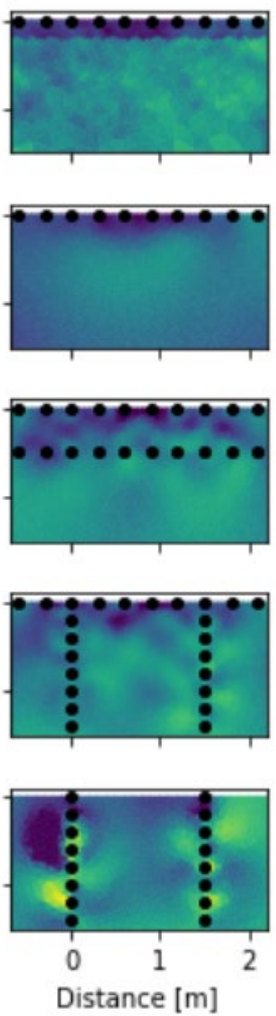

DOY 182
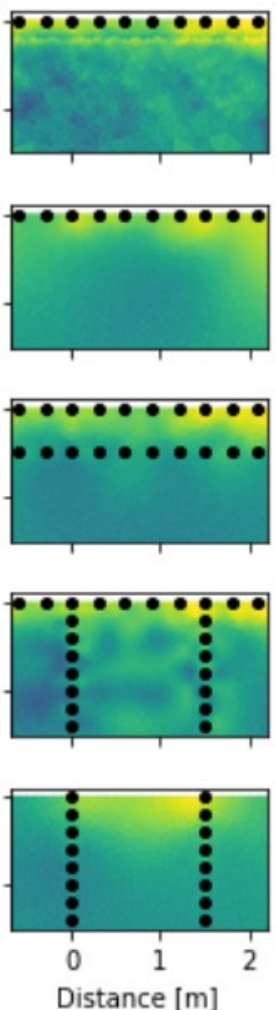

DOY 212
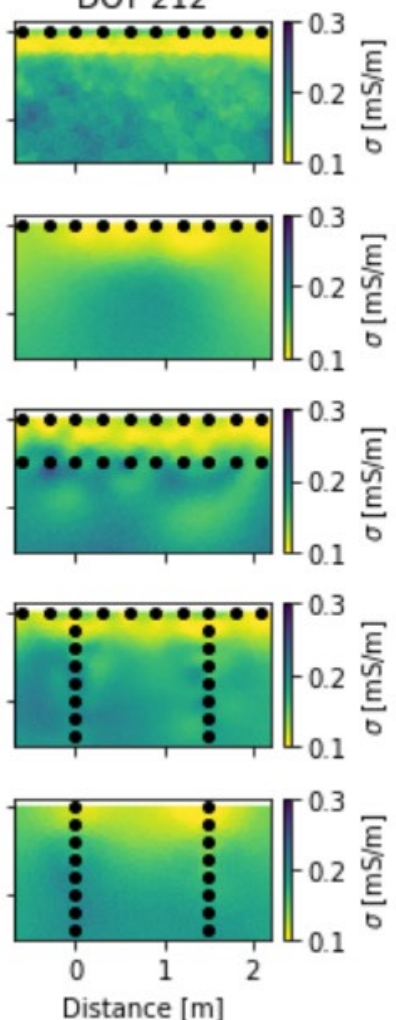

Figure 2 Recovered resistivity distributions for the four electrode configurations investigated (TRAN, TRAN_BUR, TRAN_BORE, BORE) at the four selected timeframes. The first row shows the 'true' resistivity distribution (TRUE) generated with Hydrus and the selected petrophysical relationships.

\section{Conclusions}

Electrical resistivity tomography has potential to monitor soil moisture depletion patterns driven by root water uptake. A combination of surface and subsurface electrodes is thereby indispensable. Nevertheless, a lot of uncertainty remains in field conditions. Some can be alleviated by careful site characterization (eg. heterogeneity of hydraulic characteristics) and independent measurements of influencing factors (eg. temperature, salinity, soil moisture). Others are inherent to the method and need to be taken into account during data interpretation.

\section{References}

Atkinson, J.A., M.P. Pound, M.J. Bennett, and D.M. Wells. 2019. "Uncovering the Hidden Half of Plants Using New Advances in Root Phenotyping." Current Opinion in Biotechnology 55: 1-8. https://doi.org/10.1016/j.copbio.2018.06.002.

Binley, A., and A. Kemna. 2005. "Electrical Methods." In Hydrogeophysics, edited by Rubin and Hubbard, 129-56. Springer.

Costa, Corrado, Ulrich Schurr, Francesco Loreto, Paolo Menesatti, and Sebastien Carpentier. 2019. "Plant Phenotyping Research Trends, a Science Mapping Approach." Frontiers in Plant Science 9. https://doi.org/10.3389/fpls.2018.01933.

Ehosioke, Solomon, Frédéric Nguyen, Sathyanarayan Rao, Thomas Kremer, Edmundo PlacenciaGomez, Johan Alexander Huisman, Andreas Kemna, Mathieu Javaux, and Sarah Garré. 2020. "Sensing the Electrical Properties of Roots: A Review." Vadose Zone Journal 19 (1): e20082. https://doi.org/10.1002/vzj2.20082.

Xie, Chuanqi, and Ce Yang. 2020. "A Review on Plant High-Throughput Phenotyping Traits Using UAV-Based Sensors." Computers and Electronics in Agriculture 178 (November): 105731. https://doi.org/10.1016/j.compag.2020.105731. 